\title{
Determination of reliability indices of the distribution network based on data from AMI
}

\author{
Mirosław Kornatka*, Anna Gawlak, and Grzegorz Dudek \\ Czestochowa University of Technology, Faculty of Electrical Engineering, 42-200 Czestochowa, \\ Poland
}

\begin{abstract}
Improving reliability of a power network is an issue of great importance for power distribution companies. The aim stated by the President of Energy Regulatory Office to improve the reliability of the distribution network includes steps towards improving the monitoring and control of the network managed by distribution companies. Ensuring continuity of energy supply is one of distribution companies' duties and one of the criteria used to assess the effectiveness of network operation. Distribution companies all over the world employ indices SAIDI, SAIFI and MAIFI to determine mean values concerning the number of time of interruptions per consumer in a year. The paper deals with methods of correct and precise determination of the values of the reliability indices SAIDI, SAIFI and MAIFI by means of the application Advanced Metering Infrastructure in the distribution network.
\end{abstract}

\section{Introduction}

During the recent decades a new concept, known as Smart Grid, has emerged in the operation of power network, . It is being widely implemented by distribution companies. The implementation of Smart Grid is intrinsically connected with the idea of Smart Metering, and the first step towards constructing modern power networks is implementing the application Advanced Metering Infrastructure (AMI). The application combines three components: measuring infrastructure, telecommunications infrastructure and a central information processing system. On-line measurement data coming from AMI offer excellent possibilities of monitoring the network, together with tools for its analysis and synthesis, which is another step towards implementing Smart City. The system AMI not only offers an on-line analysis of energy consumption and helps to take conscious decisions, but it also provides tools for determining indices of power supply continuity and for effective managements of the network in emergencies. In this way, it is useful for solving technical and organizational problems resulting from the need to identify and locate a fault in a distribution network. The system AMI identifies faults with high precision with respect to time and location, enabling a close monitoring of the distribution network operation. When events reported by AMI are correlated with those obtained from the system Supervisory Control and Data Acquisition (SCADA), a wider perspective on the

* Corresponding author: kornatka@el.pcz.czest.pl 
condition of the network is afforded and information coming from various sources can be verified.

\section{Reliability of distribution network}

Analysis of operation of the distribution network can be carried out from different perspectives; one of them is supply reliability which is the key notion in the estimation of the supply continuity and service quality [1-8]. Evaluation of the reliability of LV networks is one of the important points considered in the analysis of the distribution power system. In the case of failure in this grid, both the power distribution companies and its customers can suffer severe economic losses.

Using the indices SAIDI and SAIFI for assessing the reliability of electricity supply is a generally accepted method, applied all over the world. These indices are also used to compare the level of reliability across the power system. In Poland, according to the Ordinance of the Minister of Economy of 2007 on the specific conditions of the functioning of the power system, the power distribution companies are obliged to announce on their website the indicators concerning the time of interruptions in electricity supply calculated for the preceding calendar year.

The power distribution companies are required to make public the following reliability indices: SAIDI and SAIFI (for long-term scheduled and unplanned interruptions) and MAIFI. An interruption is classified as scheduled when network users are informed in advance, to allow for the execution of scheduled works on the distribution system. Unplanned interruption is classified as random caused by transient or permanent faults, mostly related to external events or equipment failures without notice in advance to the customers. Interruptions in electricity supply are divided into:

- short - between 1 second and 3 minutes

- long - between 3 minutes and 12 hours

- very long - between 12 and 24 hours

- disastrous - longer than 24 hours.

SAIDI is defined as follows:

System Average Interruption Duration Index (SAIDI) gives the average amount of time per year while the energy supply to a customer is interrupted. It is calculated by means of the following expression [9]:

$$
\mathrm{SAIDI}=\Sigma\left(r_{i} N_{i}\right) / N_{T}
$$

where the summation is taken over all incidents, either at all voltage levels or only at selected voltage levels; $r_{i}$ is the restoration time for each incident; $N_{i}$ is the number of customers affected by each incident; $N_{T}$ is the total number of customers in the system for which the index is calculated (expressed in minutes lost per customers per year).

SAFI is defined as follows:

System Average Interruption Frequency Index (SAIFI) corresponds to the average number of times per year while the power supply of a customer is interrupted. It is calculated by means of the following expression [9]:

$$
\mathrm{SAIFI}=\Sigma\left(N_{i}\right) / N_{T}
$$

given in number of interruptions per customer per year.

SAIDI and SAIFI are calculated including or excluding a specific cause of interruption (force majeure, exceptional events etc.) [9]. 
The expression for calculating MAIFI is the same as the one for calculating SAIFI (2), where the summation is taken over all incidents resulting in short interruptions.

\section{Advanced Metering Infrastructure}

The installation of AMI is looked upon as a bridge to the construction of Smart Grid. AMI is an integrated system of elements including intelligent meters, communication systems and modules, hubs and recorders. AMI enables bidirectional communication, by means of various media and means, between the central system and selected meters. An integrated system of this kind records and stores data on energy consumption, sends control signals to devices and carries out their remote configuration. An important functionality of the system AMI is access to events occurring in the power system, registered by meters, which act as distributed information sources. Effective information flow is afforded by a fast and reliable communication infrastructure [10].

AMI solutions should not only satisfy current needs, but they should also anticipate future challenges and developments that may appear in the power industry. The directions of such developments include increase in demand for charging stations for electric vehicles, increasing number of distributed generation stations, and increasing popularity of the Demand Response program among energy producers and consumers.

\subsection{Selected advantages of AMI}

The system AMI appears to offer a number of advantages, the most important of which are [11]:

- consumers do not have to present when a meter is being read,

- errors resulting from reading a meter are minimized,

- operational cost involved in obtaining data is minimized,

- billing is always carried out in the time scheduled on the basis of real data,

- reports can be sent frequently, e.g. once a day or once a week,

- tools for controlling the users' energy consumption, e.g. consumption limits are available,

- failures in the power network can be detected fast and without effort,

- anomalies in energy use can be identified fast,

- data can be used not only to improve billing systems but also customer service.

\subsection{Selected disadvantages of AMI}

Apart from the fact that implementing the system AMI is costly, it also has some disadvantages, including [11]:

- storing detailed data on a user's energy consumption threatens the users' privacy,

- unlicensed third parties may use the system to monitor consumption,

- some electronic elements of the AMI system are sensitive to atmospheric discharges, which may lead to complete or partial interruption if data flow between intelligent meters and the central system.

\subsection{The functionality "last gasp" in intelligent meters}

Effective outage detection and management is important to consumers. The availability of precise and timely information about outages should enable network businesses to respond to outages effectively and quickly [12]. Last gasp is a functionality that maintains power 
supply of an intelligent meter and its communication with a power network operator even when regular power supply is interrupted due to failure. Thanks to this functionality it is possible to send detailed information on events occurring in the network under all conditions.

Detecting interruptions and sending information on their occurrence can rely on a number of technological means. The functionality last gasp can be limited to supporting minimal power supply necessary for sending one short message to the network operator informing them about interruption. For such a message to reach the operator, all the intervening communication devices between an intelligent meter (i.e. source of the message) and the operator have to work correctly. Thanks to this functionality, the network operator receives a message about an interruption very fast. This, in turn, enables the operator to act immediately and fix a failure. Thus, the operator can manage failure situations effectively, as the failure identification and solution to it can be applied very fast, which is an important factor justifying the idea of smart grid. The functionality is also beneficial for consumers because it minimizes the time necessary to restore supply by registering the onset of interruption immediately. In this way, last gasp enhances the quality of service offered to customers and in consequence contributes to greater customer satisfaction. On the disadvantage side, it has to be mentioned that in the case of major failures, the amount of information received by the operator can be excessive.

\section{Method for determining the indices SAIDI and SAIFI on the basis of data obtained from AMI}

The traditional reliability calculation system using system SCADA cannot calculate the reliability indices accurately - it cannot obtain the detailed customers' power consumption and interruption data of each outage. In terms of reliability indices, an electricity user is equivalent to a meter (LO) connected at a supply point. A supply point is characterized by a set of attributes, including a name, address, geographical location, meter data, states and events. A supply point associates a real measuring system and its parameters with a location of a measurement. The measuring data and events used for analysis, aggregation, validation and estimation of the data are saved in a database environment, so it can be processed with high efficiency.

At the level of a MV/LV transformer station, balancing meters (LB) are installed. Such meters are equipped with extensive capabilities for carrying out measurements and recording such events as lowering or increasing voltage and power interruption. At the level of MV and HV, networks the system SCADA operates. It has to be noted that SCADA records events of other types than AMI: SCADA obtains information on events automatically through various kinds of telecommunications equipment, power protection control systems or disconnector positions. Part of the events recorded by SCADA are activities of an operator switching a circuit.

By means of setting parameters of the balancing meters and user meters, the sensitivity of the system AMI can be set an appropriate level, for instance, the system may ignore micro-interruptions. Because of that the number of events registered (ei in fig. 1) by user meters (LO), balancing meters (LB) at MV/LV transformer stations and SCADA may be different, as illustrated graphically in Fig.1. 


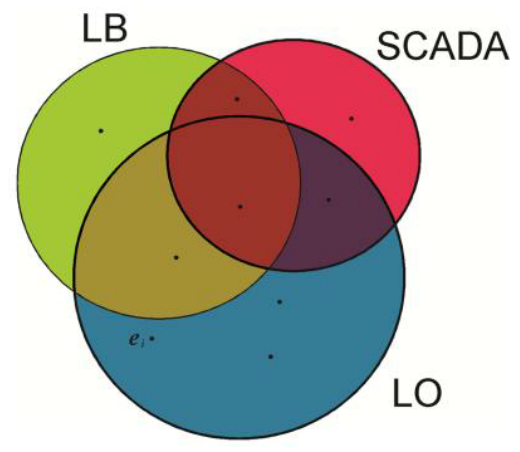

Fig. 1. Correlation of event spaces registered by SCADA as well as balancing meters (LB) and user meters (LO).

The greatest number of bits of information is registered by user meters (LO). Attributing a user meter to a network sequence and a LV/MV transformer provides information which is not registered by a balancing meter. Events registered by SCADA include a different set of data, such as whether an interruption was planned or occurred due to failure. Besides, SCADA delivers information on the cause of an interruption. When determining the SAIDI and SAIFI indices it is therefore necessary to correlate the events registered at different level and to take into account their coincidence or lack thereof.

The ability to register events by intelligent meters is a functionality dependent both on the meter's hardware installed by a manufacturer and on setting of the parameters by an operator. Meters typically register such events as Primary Power Down, Primary Power Up, Voltage Sag, Voltage Swell, Phase Loss, Magnetic Tamper, Cover Removed, Measurement Error, Load Disconnect State Changed, Configuration Error.

To analyze events from AMI meters and balancing meters, it is necessary to develop a procedure for calculating the duration of a power interruption. The first stage in determining the reliability indices is selecting the events "power down" and "power up", i.e. representing an onset and termination of an interruption. The next stage is an aggregation of data, during which it is necessary to consider replacements of user meters and balancing meters (dates of assembly and disassembly) and changes in the network structure.

Table 1 presents a sequence of "Primary power down" and "Primary power up" events for a METER_ID.

Table 1. A sequence of events "Primary Power Down" and "Primary Power Up" for a selected meter.

\begin{tabular}{|c|c|c|c|}
\hline METER_ID & EVENT_DATE & EVENT_TIME & CODE_ID \\
\hline 38709677 & 10.01 .2018 & $01: 00: 43$ & D3 \\
\hline 38709677 & 10.01 .2018 & $01: 10: 31$ & U3 \\
\hline 38709677 & 14.02 .2018 & $18: 00: 47$ & D3 \\
\hline 38709677 & 14.02 .2018 & $18: 10: 34$ & U3 \\
\hline 38709677 & 15.02 .2018 & $15: 20: 47$ & D3 \\
\hline 38709677 & 15.02 .2018 & $15: 30: 34$ & U3 \\
\hline 38709677 & 14.03 .2018 & $23: 4242$ & D3 \\
\hline 38709677 & 15.03 .2018 & $00: 10: 29$ & U3 \\
\hline
\end{tabular}

where: D3 - Primary Power Down, U3 - Primary Power Up.

The number of users affected by the failure is equal to the number of supply points in the LV circuits. The number of users to which the index refers (i.e. the denominator in formulas 1 and 2) is the number of end users connected to the operator's grid as of December in the year preceding the calculations. 
According to legal regulations, each elementary event is to be stored as a separate position in the distribution company's register and has to be identifiable at least by the following:

- identification marks of the MV/LV station and the number of a transformer (if there is more than one),

- the number of users affected by interruption due to switching off a transformer or transformers, if there is more than one in a station,

- the number of users supplied from a MV/LV station or a MV/LV transformer,

- interruption duration (the date and time of onset and the date and time of termination).

Input data for the procedure include events registered by AMI meters and balancing meters. Output data are duration times of interruptions on particular meters divided into long, very long and disastrous.

The interruption durations registered by user meters and balancing meters are utilized for calculating the values of SAIDI and SAIFI for a section and a MV/LV network with consideration of disastrous interruptions and without consideration of disastrous interruptions, respectively, and also with the division into planned and unplanned interruptions. According to the algorithm of calculating SAIDI and SAIFI, every interruption lasting above 3 minutes is taken into account, including interruptions caused by switching the power on and off in the network.

Interruptions can be classified as scheduled, according to regulations, only if they were planned and announced in due advance and do not exceed the duration announced to users. All other interruptions are considered as unscheduled. If an interruption is terminated later than planned or if it begins before the time scheduled, the durations of an interruption exceeding the scheduled time are considered as unscheduled.

\section{Reliability analysis of the distribution network based on data from AMI - a case study}

A real distribution system is used for the analysis. The analyzed network supplies over 2 thousand customers $\left(N_{T}\right)$ using $17 \mathrm{MV} / \mathrm{LV}$ transformer stations. The network is fully equipped with the AMI infrastructure, both at consumer meters and at MV/LV transformer stations. The analysis presented below is based on 105.000 events registered by user meters (LO) and over 400 events registered by balancing meters (LB) in the period of one year. In that period the system SCADA registered 148 events in the area of MV network under scrutiny.

To determine the SAIDI, SAIFI and MAIFI indices on the basis of events registered by the AMI infrastructure, it is of key importance to interpret these events correctly, together with their interdependences and time correlations. Thus, events registered by balancing meters have to be tied with events registered by SCADA and those from consumer meters (Fig. 1). All events registered by SCADA should be recorded in the system, including those that are not reflected in events registered by balancing meters. Determining interdependences between events registered by various sources is necessary for obtaining real values of SAIDI and SAIFI for particular MV/LV stations and particular consumers.

Besides, it has to be taken into account in the analysis whether any of the LB or LO meters have been replaced. This is significant for determining the number of consumers connected to a MV/LV station. 


\subsection{Correlation algorithm for unscheduled and scheduled interruptions registered by SCADA with interruptions registered in LB/LO}

To correlate electricity interruptions registered by SCADA with interruptions in MV/LV stations registered in balancing meter event files, it is necessary to take into account that these two kinds of events may not be synchronized - see Fig. 2.

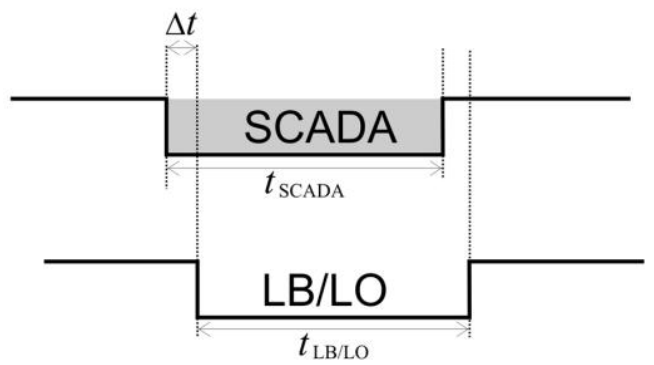

Fig. 2. Correlation between events registered in SCADA with LB- and LO-registered events.

The parameter $\Delta t$ defines the maximal time lapse between an onset of an interruption registered by SCADA and an onset of a voltage interruption event received from LB and LO. Correlations of all events were carried out with the parameter $\Delta t$ equal to $2 \mathrm{~s}$.

Another criterion parameter is $\Delta l$, defining the absolute value of the maximal difference between the duration of interruptions as registered by SCADA and the duration obtained from LB and LO. This parameter is expressed as

$$
\Delta l=\left|t_{\mathrm{SCADA}}-t_{\mathrm{LB} / \mathrm{LO}}\right|
$$

and in the calculations its value was assumed as $\Delta l=0.1 \mathrm{~s}$.

To obtain correlation between the scheduled interruptions registered by SCADA and by balancing meters it is also necessary

- to define scheduled interruption in SCADA,

- to define the duration of a scheduled interruption in SCADA and to register the real time of interruption in this system.

Precise information on the cause of interruptions is available in SCADA. On the basis of data obtained from SCADA and the balancing meters and user meters, it is possible to precisely delineate the area affected by the interruption by analyzing events occurring in neighboring stations. When a number of stations are not working at the same time, it may be a sign of a breakdown or of scheduled maintenance works in the distribution network.

\subsection{Statistical analysis of SAIDI and SAIFI indices for unscheduled interruptions at MV/LV stations}

Statistical metrics of random variables presented in the form of a table do not always reveal all the required information on the population under scrutiny. Presenting the estimated statistical values in the form of a graph, such as boxplot, may offer a much more lucid way of viewing them.

The results of calculations of SAIDI and SAIFI for the $17 \mathrm{MV} / \mathrm{LV}$ transformer stations are presented in graphs (Figs 3-4). The calculations were performed for various sets of source data representing various events $\left(e_{i}\right)$, i.e. those obtained from SCADA, from the balancing meters and user meters as well as combination of them. 


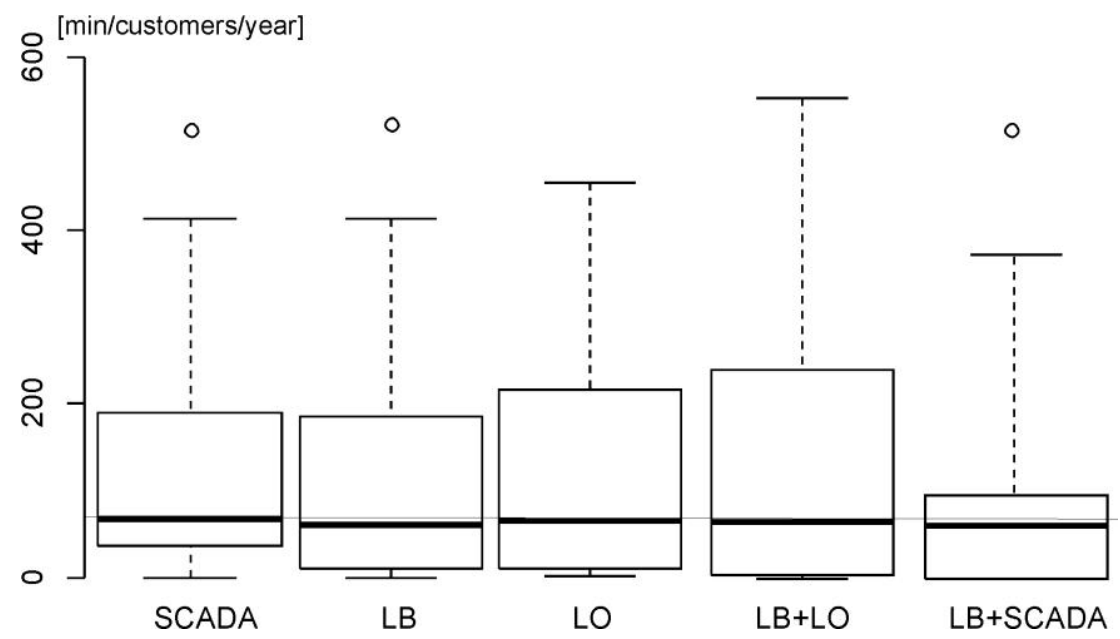

Fig. 3. Boxplot of the SAIDI values of unscheduled events at MV/LV transformer station obtained on the basis of events registered by SCADA, LB, LO, LB+LO and LB+SCADA.

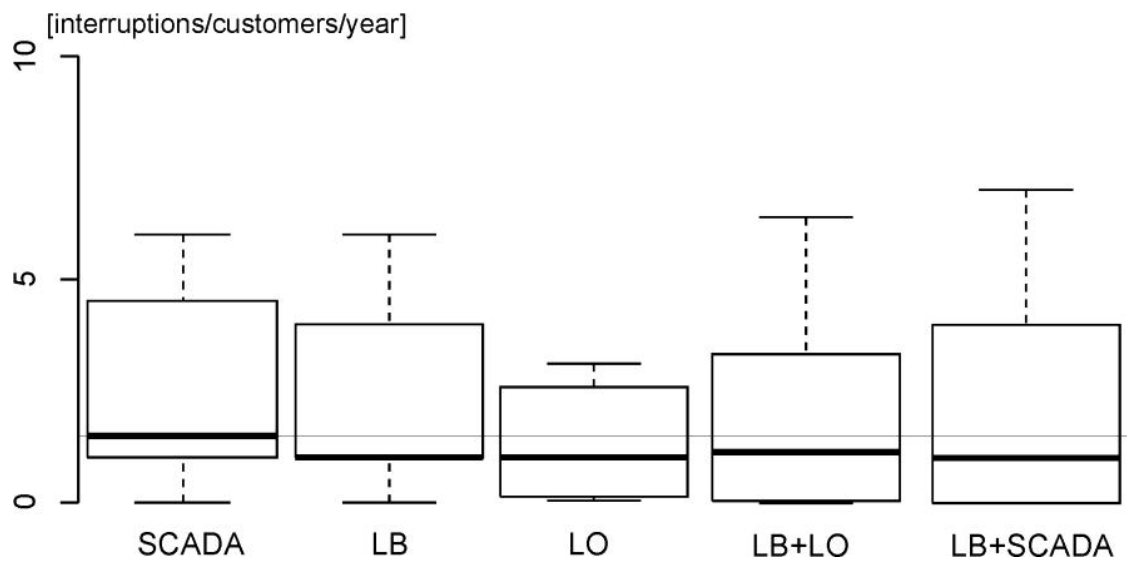

Fig. 4. Boxplot of the SAIFI values of unscheduled events at MV/LV transformer station obtained on the basis of events registered by SCADA, LB, LO, LB+LO and LB+SCADA.

The differences visible in the graphs are due to the fact that the LO data include detailed information on interruptions occurring at users' premises, which are not registered by LB or SCADA. Other differences stem from distinct methods of parameterization of LO and LB meters as well as from the specific way of registering events by SCADA.

\section{Conclusions}

The system AMI is a fundamental component of Smart Grid, by means of which it is possible to transmit information on events occurring within the network. The system AMI is therefore an important element in the construction of the system SCADA in the LV network. 
The system AMI provides highly accurate data for precise determination of reliability indices for particular users, LV network sections, MV/LV transformer stations and MV network sections.

The functionalities of the system AMI and its efficiency are a key factor enabling the recording of events within the LV network by means of intelligent meters.

The above-presented analysis of SAIDI and SAIFI obtained on the basis of events registered by the AMI system brings to the fore a number of issues that have to be taken into account in calculating these indices. The data available from the systems AMI and SCADA significantly facilitate the systematic scrutiny of the power system, which includes precise calculation of the reliability indices. Besides, the system AMI offers other useful functionalities, such as localizing the premises of interruption.

\section{References}

1. R. Billinton and R. Allan, Reliability Evaluation of Power Systems, 2nd ed., (New York: Plenum Press, 1996)

2. R. E Brown, Electric Power Distribution Reliability, 2nd ed., (CRC Press, 2009)

3. K. Moslehi and R. Kumar, "Smart Grid - a reliability perspective" in Proc. Innovative Smart Grid Technologies (ISGT 2010) pp. 1-8. (2010)

4. R. Briš, P. Byczanski, R. Goňo and S. Rusek, "Discrete maintenance optimization of complex multi-component systems," Reliability Engineering \& System Safety, vol. 168, pp. 80-89, (2017)

5. T. Pydych, M. Szydłowski and J. Sowiński, "Forecast of Power Generation and Heat Production from Renewable Energy Sources," in Proc. 2016 Energy and Fuels, E3S $W e b$ of Conferences vol. 14, article number 01012, DOI: https://doi.org/10.1051/e3sconf/20171401012 (2017)

6. M. Kornatka, A. Gawlak, „Comparative Analysis of Operating Conditions in Polish Medium-voltage and $110 \mathrm{kV}$ Networks" in Proc. 8th International Scientific Symposium on Electrical Power Engineering, pp. 57-60 (2015)

7. M. Kornatka, "The weighted kernel density estimation methods for analysing reliability of electricity supply" in Proc. 17th International Scientific Conference on Electric Power Engineering, pp. 2-5 (2016)

8. M. Kolcun, M. Kornatka, A. Gawlak and Z. Čonka, "Benchmarking the reliability of medium-voltage lines," Journal of Electrical Engineering vol. 68 (3), pp. 212-215, (2017)

9. 6th CEER Benchmarking Report on the Quality of Electricity and Gas Supply. Brussels: CEER, [Online]. Available: https://www.ceer.eu/ (2016)

10. S. W. Luan J. H. Teng S. Y. Chan and L. C. Hwang, "Development of an automatic reliability calculation system for advanced metering Infrastructure" in Proc. 8th IEEE International Conference on Industrial Informatics (INDIN 2010), pp. 342-347 (2010)

11. K. Billewicz, Smart Grids. Inteligentne sieci elektroenergetyczne, Radom: IMD, (2015)

12. Smart Metering Implementation Programme: Response to Prospectus Consultation Supporting Document 3 of 5 Design Requirements, [Online]. Available: https://www.ofgem.gov.uk/sites/default/ files/docs/2011/03/design-requirements_0.pdf (2011) 